\title{
Intensive Care Unit Utilization Among Medicare Patients Hospitalized with Non-ST-Segment Elevation Myocardial Infarction
}

\author{
Alexander C Fanaroff ${ }^{a, b}$, Eric D Peterson ${ }^{a, b}$, Anita Y Chen ${ }^{b}$, Laine Thomas ${ }^{b}$, Jacob D Dollc, \\ Christopher B Fordyce ${ }^{a, b}$, L Kristin Newby ${ }^{a}, b$, Ezra A Amsterdam ${ }^{d}$, Mikhail N Kosiborod ${ }^{\mathrm{e}}$, \\ James A de Lemos ${ }^{f}$, and Tracy $Y$ Wanga,b \\ aDivision of Cardiology, Duke University, Durham, NC \\ bDivision of Cardiology, Duke University, Durham, NC \\ 'Division of Cardiology, VA Puget Sound Health System and the University of Washington, \\ Seattle, Washington \\ dDivision of Cardiology, University of California (Davis), Sacramento, CA \\ eDivision of Cardiology, Mid-America Heart Institute, Kansas City, KS \\ fDivision of Cardiology, University of Texas - Southwestern, Dallas, TX
}

\section{Abstract}

Importance-Intensive care unit (ICU) utilization may have important implications for the care and outcomes of patients with non-ST-segment elevation myocardial infarction (NSTEMI).

Objectives-To examine inter-hospital variation in ICU utilization in the United States for older adults with hemodynamically stable NSTEMI and outcomes associated with ICU utilization among patients with at low, moderate, or high mortality risk.

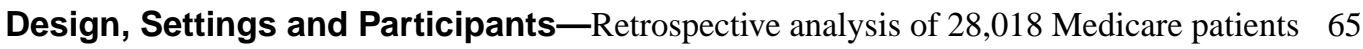
years old admitted with NSTEMI to 346 hospitals participating in ACTION Registry-GWTG between April 1, 2011 and December 31, 2012. Patients with cardiogenic shock or cardiac arrest on presentation were excluded.

Exposure-Hospitals with high (>70\% NSTEMI patients treated in an ICU during the index hospitalization), intermediate (30-70\%), or low $(<30 \%)$ ICU utilization rates

\section{Main Outcome and Measure-30-day mortality}

Results-Of NSTEMI patients $\geq 65$ years old, 11,934 (43\%) had an ICU stay. The proportion of NSTEMI patients treated in the ICU varied across hospitals (median 38\% [26\%,54\%]), but there were no significant differences in hospital characteristics or NSTEMI patient characteristics

Corresponding author: Tracy Y. Wang, MD, MHS, MSc, Duke Clinical Research Institute, 2400 Pratt Street, Durham, NC 27705, Phone: 919-668-4684, Fax: 919-668-7057, tracy.wang@dm.duke.edu.

Author Contribution: Drs. Fanaroff and Wang had full access to all of the data in the study and takes responsibility for the integrity of the data and the accuracy of the data analysis

Conflict of Interest Disclosures: All other authors report no conflicts of interest relevant to this presentation. 
between hospitals with high, intermediate, or low ICU utilization rates. Compared with high ICU utilization hospitals, hospitals with low or intermediate ICU utilizations rates were only marginally more selective of higher risk patients, as determined by ACTION in-hospital mortality risk score or initial troponin level. Thirty-day mortality rates did not significantly differ based on hospital ICU utilization (high vs. low: $8.7 \%$ vs. $8.7 \%$, adjusted OR $0.91,95 \%$ CI $0.76-1.08$; intermediate vs. low: $9.6 \%$ vs. $8.7 \%$, adjusted OR $1.06,95 \%$ CI $0.94-1.20$ ). The relationship between hospital ICU utilization and mortality was similar in analyses stratified by low, moderate, or high ACTION risk score categories (adjusted interaction $p 0.86$ ).

Conclusions and Relevance-ICU utilization for older NSTEMI patients varied significantly among hospitals. This variability was not explained by hospital characteristics nor driven by patient risk. Post-MI mortality did not significantly differ among hospitals with high, intermediate, or low ICU utilization.

\section{INTRODUCTION}

In the United States, 450,000 patients are admitted to the hospital with a non-ST-segment elevation myocardial infarction (NSTEMI) annually. ${ }^{1}$ Guidelines recommend that these patients be managed in an intensive care unit (ICU), coronary care unit (CCU) or monitored bed, ${ }^{2}$ and recent studies suggest significant variability in ICU utilization. ${ }^{3-5}$ Some hospitals routinely admit patients with NSTEMI to the ICU while others may attempt to select patients at risk of clinical deterioration for ICU admission.

Location of care may have important implications. A recent study of Medicare beneficiaries diagnosed with pneumonia found that ICU care was associated with lower adjusted mortality. ${ }^{6}$ While ICU care has not been associated with reduced mortality in all-comer MI patients, ${ }^{4}$ whether hemodynamically stable but higher risk patients specifically derive benefit has not been tested. It is also uncertain whether hospitals effectively triage NSTEMI patients at higher mortality risk to the ICU.

The National Cardiovascular Data Registry ${ }^{\circledR}$ (NCDR) Acute Coronary Treatment and Intervention Outcomes Network Registry ${ }^{\circledR}$ - Get With the Guidelines ${ }^{\mathrm{TM}}$ (ACTION Registry - GWTG) collects data for consecutive MI patients treated in routine practice in the United States (US), and is unique in capturing sufficient clinical information to calculate predicted mortality risk on admission. Using this registry, linked to Center for Medicare and Medicaid Services (CMS) claims records to provide information on ICU utilization, we aimed to (1) describe current practice patterns and inter-hospital variability in ICU utilization for older NSTEMI patients, (2) determine whether hospitals selectively treated older patients at higher mortality risk in the ICU, and (3) examine the association of hospital ICU utilization rates with 30-day mortality based on patients' mortality risk on admission.

\section{METHODS}

\section{Patient population}

The ACTION Registry - GWTG is a quality improvement registry that captures consecutive patients admitted to participating hospitals with STEMI and NSTEMI. ${ }^{7,8}$ Trained data abstractors at each hospital collect detailed information on medical history, clinical 
presentation, and in-hospital treatment via retrospective chart review. Real-time data quality feedback and annual audits ensure data accuracy. Patients $\geq 65$ years old, in ACTION Registry - GWTG have previously been linked to their CMS claims data using 5 indirect identifiers (date of birth, sex, hospital identifier, date of admission, and date of discharge). 9-11 We used this linked data source to determine ICU utilization and post-discharge outcomes.

Of 44,915 NSTEMI patients in the linked database between April 1, 2011 and December 31, 2012 , we excluded 12,433 patients who initially presented to hospitals that do not participate in ACTION Registry - GWTG, and were transferred from those hospitals into ACTION Registry - GWTG hospitals, as the triaging decision- for these patients depend on hospital policies, provider preference, variations in the amount of information conveyed prior to transfer, and patient characteristics at the time of transfer that introduce substantial risk of unmeasured confounding. We excluded patients with cardiac arrest $(n=688)$ or cardiogenic shock $(n=473)$ on admission, as these patients had a compelling reason for ICU admission. For patients with multiple MI admissions during the study period $(n=1,348)$, follow-up began at the start of the first admission. Finally, in order to ensure reasonable precision of hospital-specific ICU utilization rates, 153 hospitals admitting <25 MI patients over the study period were excluded $(n=1,955)$. To ensure that hospitals reporting $0 \%$ ICU utilization for patients with NSTEMI had ICU capability, we cross-referenced the ACTION Registry GWTG database with data from the American Hospital Association survey; all hospitals reporting $0 \%$ ICU utilization had ICU beds. Our final study population included 28,018 Medicare patients treated for NSTEMI at 346 US hospitals.

\section{Statistical Analysis}

Patients with revenue center codes for ICU or CCU utilization (see eAppendix 1) during the index MI hospitalization were classified as having been treated in an ICU. After examination of the distribution curve, we divided hospitals into 3 groups: high ICU utilization (>70\% of all patients with NSTEMI treated in the ICU), intermediate ICU utilization (30-70\%), and low ICU utilization $(<30 \%)$. We compared hospital features, patient characteristics and treatment patterns during the index hospitalization of NSTEMI patents treated at high-, intermediate-, and low-ICU utilization hospitals. Categorical variables were reported as frequencies with percentages and compared using $\chi^{2}$ tests, and continuous variables were reported as medians with $25^{\text {th }}$ and $75^{\text {th }}$ percentiles and compared using Kruskal-Wallis tests.

To determine whether hospitals were selectively treating patients at higher risk of in-hospital complications in the ICU, we calculated the ACTION in-hospital mortality risk score (ACTION risk score) for each patient. ${ }^{12}$ The ACTION risk score was derived and validated in a population of patients with MI, and predicts in-hospital mortality (c-statistic 0.84 ) based on variables present on presentation: age, baseline serum creatinine, systolic blood pressure, baseline troponin ratio ( $\times$ upper limit of normal [ULN]), heart rate, presence of heart failure or shock, electrocardiographic changes, and prior peripheral arterial disease. Based on cutpoints defined in the initial derivation of the ACTION risk score, we divided patients into groups with ACTION risk score $>40,30-40$, and $<30$, corresponding to high, moderate, and low predicted in-hospital mortality risk. 
Box plots of ACTION risk score, as a continuous variable, were displayed for patients with and without an ICU stay across hospital ICU utilization categories. We then compared the proportion of patients with ACTION risk score $>40,30-40$, and $<30$ treated in an ICU among high-, intermediate-, and low-ICU utilization hospitals using a $\chi^{2}$ test. We repeated this analysis dividing patients into different risk categories based on their initial troponin levels: $\unlhd \times$ institutional troponin assay upper limit of normal (ULN), $1-10 \times \mathrm{ULN}$, and $>10$ $\times$ ULN.

We compared unadjusted 30-day mortality from the time of index admission among hospitals with high, intermediate, or low ICU utilization rates. This was first reported among all NSTEMI patients, and then stratified by ACTION risk score. We used logistic generalized estimating equations regression with an exchangeable working correlation matrix to account for within-hospital clustering, and to adjust for patient and hospital characteristics. ${ }^{13}$ Odds ratios (OR) and 95\% confidence intervals (CI) were reported with low ICU utilization hospitals as the reference group. The model adjusted for patient-level covariates from the ACTION Registry - GWTG mortality risk model ${ }^{12}$ and hospital-level covariates (variables listed in eAppendix 2). Furthermore, we tested the interaction between hospital ICU utilization rate and patient ACTION risk score stratum as it relates to 30-day mortality. We repeated this analysis treating hospital-level ICU utilization as a continuous variable, and reported OR and $95 \%$ confidence intervals for every $10 \%$ increase in ICU utilization.

To account for our inability to determine whether patients were directly admitted to the ICU or transferred to the ICU after sustaining an in-hospital complication and to minimize the influence of unmeasured illness severity that biases towards ICU utilization, we performed a sensitivity analysis excluding patients with any documented complication mandating ICU admission during the index hospitalization. This included in-hospital development of cardiogenic shock, cardiac arrest, or stroke as captured by the ACTION Registry-GWTG data collection form, as well as patients with diagnosis or procedure codes that mandate ICU-level care: those indicating hemodynamic compromise requiring inotrope/vasopressor or mechanical support, need for cardiopulmonary resuscitation, arrhythmia requiring pacing, mechanical complication post-MI, respiratory failure, stroke, or intracranial hemorrhage (see eAppendix 3). We repeated the above analyses examining hospital rate of ICU utilization and association with 30-day mortality after excluding these patients.

We observed low rates of missing data: less than $1 \%$ for all variables except BMI (1.1\%) Missing values in continuous covariates were imputed to the sex-specific median of the nonmissing values. For categorical variables, missing values were imputed by the most frequent group. All statistical analyses were performed using SAS software (version 9.4, SAS Institute). The Duke Clinical Research Institute conducted all analyses.

\section{RESULTS}

\section{Rates of ICU Utilization}

Among 28,018 NSTEMI patients > 65 years old who presented to the hospital without evidence of cardiogenic shock or cardiac arrest, $43 \%$ received care in an ICU, and the 
median length of ICU stay was $2\left(25^{\text {th }}, 75^{\text {th }}\right.$ percentiles: 1,4$)$ days. ICU utilization varied substantially across hospitals (Figure 1); the median proportion of NSTEMI patients treated in an ICU was $38 \%(26,54)$ with a range from 1.9 to $100 \%$.

We classified 110 hospitals that treated $<30 \%$ of NSTEMI patients in an ICU as low ICU utilization hospitals, 193 hospitals that treated $30-70 \%$ of patients in an ICU as intermediate ICU utilization hospitals, and 43 hospitals that treated $>70 \%$ of NSTEMI patients in an ICU as high ICU utilization hospitals. Median hospital size was $317(212,452)$ beds; $82 \%$ had both PCI and cardiac surgery capabilities. There were no statistically significant differences in hospital characteristics between low, intermediate, and high ICU utilization hospitals (Table 1).

\section{Predicted Risk and ICU Utilization}

Characteristics of patients admitted to high, intermediate, and low ICU utilization hospitals are presented in Table 1 . The median patient age was $77(71,84)$ years; $53 \%$ were men and $86 \%$ were white. Differences in clinical characteristics, including comorbid illnesses, severity of illness on presentation, and other markers of MI severity (such as peak troponin and ejection fraction) of NSTEMI patients treated at high, intermediate, and low ICU utilization hospitals were statistically significant, although differences may be too small to be clinically meaningful.

The median ACTION risk score was $33(27,40) ; 34 \%$ of patients had an ACTION risk score $<30,44 \%$ had an ACTION risk score 30-40, and 23\% had ACTION risk score >40. The distribution of ACTION risk scores was similar among NSTEMI patients treated at high, intermediate, and low ICU utilization hospitals. Observed in-hospital mortality was $1 \%$ for patients with ACTION risk score <30, 3\% for those with ACTION risk score 30-40, and $11 \%$ for those with ACTION risk score $>40$. We observed statistically significant but very small differences in median ACTION risk score between patients with and without ICU stay, regardless of hospital-level ICU utilization (Figure 2). At low and intermediate ICU utilization hospitals, the median ACTION risk score for a patient not treated in an ICU was 33, compared to 34 for patients treated in the ICU.

In low and intermediate ICU utilization hospitals, patients with ACTION risk scores $>40$ were more likely than those with lower ACTION risk scores to receive ICU care; this pattern was not evident at high ICU utilization hospitals (Figure 3a). Patients with admission troponin level $>10 \times \mathrm{ULN}$ were significantly more likely to receive care in an ICU than those with lower admission troponin levels at all hospitals, but the difference was larger at low and intermediate ICU utilization hospitals (Figure $3 \mathrm{~b}$ ). Only $53 \%$ and $26 \%$ of patients with an admission troponin $>10 \times \mathrm{ULN}$ received care in the ICU at intermediate and low ICU utilization hospitals, respectively. In high ICU utilization hospitals, $>85 \%$ of patients received care in the ICU regardless of initial troponin.

\section{ICU Utilization, In-hospital Treatment and 30-Day Mortality}

There were statistically significant differences in treatment with certain guidelinerecommended medications and use of revascularization procedures at low ICU utilization hospitals compared with intermediate and high ICU utilization hospitals; however, the 
absolute differences between groups in the proportion of patients treated with any medication or procedure were small $(<3 \%)$ (Table 1). Median length of hospital stay was slightly shorter at low ICU utilization hospitals compared with intermediate and high ICU utilization hospitals ( 3 vs. 4 and 4 days). Median ICU length of stay was longer at high ICU utilization hospitals compared with low and intermediate ICU utilization hospitals (3 vs. 2 and 2 days).

Within 30 days of the index admission, 2460 (9.2\%) patients died. Unadjusted 30-day mortality was $8.7 \%$ at low ICU utilization hospitals, $9.6 \%$ at intermediate ICU utilization hospitals, and $8.7 \%$ at high ICU utilization hospitals; these rates were significantly different ( $p=0.04$ ). After adjusting for hospital characteristics and differences in patient case mix, hospitalization at a high or intermediate ICU utilization hospital versus a low ICU utilization hospital was not associated with significant differences in 30-day mortality (OR 0.91, 95\% CI 0.76-1.08 for high vs. low; OR 1.06, 95\% CI 0.94-1.20 for intermediate versus low). The relationship between hospital ICU utilization and mortality did not change when considered among patients with ACTION risk scores $>40,30-40$, and $<30$ (adjusted interaction $p=0.86$ ). When the effect of ICU utilization on mortality was evaluated as a continuous variable, increasing ICU utilization was not significantly associated with unadjusted (OR $1.01,95 \%$ CI $0.99-1.04$ ) or risk-adjusted mortality (OR 1.00, 95\% CI 0.97-1.02) per 10\% increase in ICU utilization.

\section{Sensitivity Analysis}

Overall, 4,396 patients (15.7\%) had at least 1 documented complication requiring ICU-level care - $1183(13.3 \%)$ at low ICU utilization hospitals, $2621(16.8 \%)$ at intermediate ICU utilization hospitals, and $592(16.7 \%)$ at high ICU utilization hospitals (eTable 1). After exclusion of these patients, $36 \%$ of NSTEMI patients were treated in an ICU. There remained inter-hospital variability in the proportion of patients treated in an ICU (eFigure 1); hospitals treated a median of $30 \%$ of these uncomplicated NSTEMI patients in the ICU $(19 \%, 47 \%)$. Patients with ACTION risk score $>40$ were no more likely to be admitted to the ICU than those with ACTION risk score 30-40 or <30 at any hospital, regardless of ICU utilization rate (Figure 4a). Among the subgroup without in-hospital complications, patients with initial troponin values $>10 \times \mathrm{ULN}$ were significantly more likely that those with initial troponin values $1-10 \times$ ULN or $\leq \times$ ULN to be treated in the ICU at low and intermediate ICU utilization hospitals (Figure 4b). In this subgroup without complications, 30-day mortality again did not differ between patients treated at high $(5.5 \%)$, intermediate $(5.4 \%)$, and low (5.1\%) ICU utilization hospitals (adjusted OR 1.02, 95\% CI 0.82-1.27 for high vs. low; adjusted OR $1.03,95 \%$ CI $0.88-1.21$ for intermediate versus low).

\section{DISCUSSION}

In this nationwide study, we found that $43 \%$ of older patients hospitalized with NSTEMI received care in the ICU in the absence of cardiogenic shock or cardiac arrest on admission, with considerable inter-hospital variability in ICU utilization. The proportion of NSTEMI patients treated in the ICU did not appear to be explained by differences in patient case mix or hospital type. The difference in ACTION risk score between patients treated with and 
without ICU care was small, regardless of whether a hospital routinely or selectively utilizes ICUs for MI patients. There was no difference in adjusted 30-day mortality between NSTEMI patients treated at high or intermediate vs. low ICU utilization hospitals, even among patients at higher in-hospital mortality risk.

Prior studies, conducted using administrative data sources also demonstrated variability in ICU utilization for MI patients, and showed no association between hospital-level ICU utilization and mortality. ${ }^{3-5}$ The NCDR collected data on each patient's MI type, hemodynamic status, troponin level, and other covariates allowing for the calculation of mortality risk on admission; thus it extends these prior findings by offering a unique perspective of how illness severity may factor into ICU utilization. While the majority of STEMI patients $(80 \%)$ are treated in an ICU, ${ }^{4}$ hospitals varied substantially in the location of care for older NSTEMI patients. There were no significant differences in hospital characteristics (including size, teaching status, and revascularization capability) across high, intermediate, and low ICU utilization hospitals. Differences in ICU utilization between hospitals also did not appear to be explained by patient case mix. Importantly, the wide inter-hospital variation in ICU utilization rates persisted after excluding patients with a documented indication for ICU care.

High ICU utilization hospitals are likely to routinely triage older NSTEMI patients to the ICU, resulting in $>70 \%$ of these patients having at least a 1-day ICU stay. As expected, the predicted mortality of ICU-treated patients was quite similar to that of non-ICU-treated patients at these hospitals. In intermediate and low ICU utilization hospitals, providers selectively triaged patients to the ICU, resulting in $30-70 \%$ and $<30 \%$ of these patients receiving care in the ICU, respectively. Yet, at these hospitals, higher risk patients were only slightly more likely to receive care in the ICU; the difference in median ACTION risk scores between patients treated in and outside of an ICU was very small. Providers may not routinely calculate a mortality risk score to guide ICU triaging. However, the ACTION risk score, like other mortality risk scores (e.g., GRACE or TIMI), ${ }^{14,15}$ is essentially a synthesis of clinical variables that providers might use to determine illness severity or project patient risk. ${ }^{12}$ Alternatively, providers might stratify risk simply based on initial degree of troponin elevation. When patients were stratified by troponin levels, low and intermediate ICU utilization hospitals still did not appear to be triaging patients with higher troponin levels to the ICU.

The results of our analysis suggest that ICU triage is largely driven by local practices and provider preference. In that light, it is unsurprising to observe no difference in 30-day mortality between NSTEMI patients treated at high or intermediate vs. low ICU utilization hospitals. The lack of mortality benefit from treatment at a high ICU utilization hospital may stem from the low likelihood of developing a life-threatening post-MI complication mandating ICU care in the modern era. ${ }^{16-18}$

These findings have important implications for decision-making with regard to location of care for initially stable NSTEMI patients. Though our data did not allow for the examination of hospital charges related to ICU care versus care in a non-ICU ward, prior studies have found a difference of $>\$ 3500$ per day. ${ }^{19}$ With almost half of the 450,000 NSTEMI patients 
treated each year in the United States being admitted to the ICU for a median stay of 2 days, expenditures on ICU care alone in NSTEMI may exceed $\$ 1.2$ billion annually. Though a relationship between volume and outcome has been demonstrated in many conditions, a recent study failed to identify a relationship between ICU MI volume and outcomes. ${ }^{20} \mathrm{~A}$ more selective ICU utilization strategy may be preferable for cost reasons; however, some initially stable patients will ultimately require ICU care, and no risk model has been developed to identify these patients prior to clinical deterioration.

Several study limitations need to be acknowledged. Our study could not determine whether patients were triaged electively to the ICU or transferred after clinical deterioration; therefore, we performed a sensitivity analysis to exclude all patients who developed a clear indication for ICU care with results similar to our primary analysis. Second, in an observational setting, we cannot infer a causal relationship between ICU utilization and mortality, nor can we exclude the possibility of unmeasured confounding. Third, our study grouped hospitals by observed ICU utilization rates but did not capture protocols hospitals use in making ICU triage decisions; ACTION Registry-GWTG also did not capture variables, such as persistent chest pain or dynamic ECG changes, which may be used in triage decisions. Finally, this analysis is limited to older patients who receive Medicare benefits and the results may not be generalizable to younger patients.

\section{CONCLUSIONS}

In this study of older patients hospitalized with NSTEMI, we found that ICU utilization was common even in uncomplicated NSTEMI patients, that there was considerable variability in hospital patterns of ICU utilization that did not appear to be explained by hospital characteristics or patient mortality risk, and that hospitals did not appear to triage patients at the highest predicted risk to the ICU. We observed no difference in 30-day mortality among NSTEMI patients treated at high or intermediate vs. low ICU utilization hospitals. Additional research is needed to identify the optimal strategy of ICU admission in patients with NSTEMI, but more judicious use of ICU admission, with a focus on identifying patients most likely to require ICU-level care, could result in similar outcomes but significant cost savings.

\section{Supplementary Material}

Refer to Web version on PubMed Central for supplementary material.

\section{Acknowledgments}

Dr. Fanaroff is supported by National Institutes of Health T32 training grant \# 5T32HL069749-13, and has received research grants through the DCRI from Gilead Sciences. Dr. Peterson has received consulting fees from Astra Zeneca, Merck, Janssen, Boehringer Ingelheim, and Bayer, and research grants from Janssen and Eli Lilly. Dr. Newby has received consulting fees from Roche Diagnostics and Philips Healthcare, and research grants from Roche Diagnostics and Abbott. Dr. Kosiborod has received consulting fees from Astra Zeneca, Eli Lilly, Amgen, Regeneron, Takeda, Edwards Lifesciences, Gilead Sciences, Roche, and Genentech, and research grants from the American Heart Association, Gilead Sciences, Genentech, Sanofi-Aventis, and Eisai. Dr. de Lemos has received consulting fees from Roche Diagnostics, Abbott Diagnostics, and Siemen's Health Care, and research grants from Roche Diagnostics and Abbott Diagnostics. Dr. Wang has received honoraria from Astra Zeneca, Eli Lilly, and Merck, and research grants through the DCRIfrom Gilead Sciences, Eli Lilly, Daiichi Sanyo, Astra Zeneca, Boston Scientific, Bristol Myers Squibb, Regeneron, and GlaxoSmithKline. 
Funding/Support: This project was supported by a grant from the Agency for Healthcare Research and Quality (U19H2O21092) to Dr. Wang.

Role of the Funder/Sponsor: The funders had no role in the design and conduct of the study; collection, management, analysis, and interpretation of the data; preparation, review, or approval of the manuscript; and decision to submit the manuscript for publication.

\section{References}

1. Mozaffarian D, Benjamin EJ, Go AS, et al. Heart disease and stroke statistics-2015 update: a report from the american heart association. Circulation. 2015; 131(4):e29. [PubMed: 25520374]

2. Amsterdam E, Wenger N. 2014 ACC/AHA guideline for the management of patients with non-STelevation acute coronary syndromes: a report of the American College of Cardiology/American Heart Association Task Force on Practice Guidelines: executive summary. Journal of the American College of Cardiology. 2014; 64:2645-2687.

3. Insam C, Paccaud F, Marques-Vidal P. The region makes the difference: disparities in management of acute myocardial infarction within Switzerland. European journal of preventive cardiology. 2014; 21(5):541-548. [PubMed: 23169865]

4. Chen R, Strait KM, Dharmarajan K, et al. Hospital variation in admission to intensive care units for patients with acute myocardial infarction. American Heart Journal. 2015; 170(6):1161-1169. [PubMed: 26678638]

5. van Diepen S, Lin M, Bakal JA, et al. Do Stable Non-ST Segment Elevation Acute Coronary Syndromes Require Admission to Coronary Care Units? American Heart Journal.

6. Valley TS, Sjoding MW, Ryan AM, Iwashyna TJ, Cooke CR. Association of Intensive Care Unit Admission With Mortality Among Older Patients With Pneumonia. JAMA. 2015; 314(12):12721279. [PubMed: 26393850]

7. Peterson ED, Roe MT, Chen AY, et al. The NCDR ACTION Registry-GWTG: transforming contemporary acute myocardial infarction clinical care. Heart. 2010; 96(22):1798-1802. [PubMed: 20736202]

8. Peterson ED, Roe MT, Rumsfeld JS, et al. A Call to ACTION (Acute Coronary Treatment and Intervention Outcomes Network) A National Effort to Promote Timely Clinical Feedback and Support Continuous Quality Improvement for Acute Myocardial Infarction. Circulation: Cardiovascular Quality and Outcomes. 2009; 2(5):491-499. [PubMed: 20031882]

9. Shah RU, de Lemos JA, Wang TY, et al. Post-hospital outcomes of patients with acute myocardial infarction with cardiogenic shock: findings from the NCDR. Journal of the American College of Cardiology. 2016; 67(7):739-747. [PubMed: 26892407]

10. Pokorney SD, Miller AL, Chen AY, et al. Implantable cardioverter-defibrillator use among Medicare patients with low ejection fraction after acute myocardial infarction. JAMA. 2015; 313(24):2433-2440. [PubMed: 26103027]

11. Hammill BG, Hernandez AF, Peterson ED, Fonarow GC, Schulman KA, Curtis LH. Linking inpatient clinical registry data to Medicare claims data using indirect identifiers. American Heart Journal. 2009; 157(6):995-1000. [PubMed: 19464409]

12. Chin CT, Chen AY, Wang TY, et al. Risk adjustment for in-hospital mortality of contemporary patients with acute myocardial infarction: The Acute Coronary Treatment and Intervention Outcomes Network (ACTION) Registry®-Get With The Guidelines (GWTG) ${ }^{\mathrm{TM}}$ acute myocardial infarction mortality model and risk score. American Heart Journal. 2011; 161(1):113-122. e112. [PubMed: 21167342]

13. Zeger SL, Liang K-Y. Longitudinal data analysis for discrete and continuous outcomes. Biometrics. 1986:121-130.

14. Antman EM, Cohen M, Bernink PJ, et al. The TIMI risk score for unstable angina/non-ST elevation MI: A method for prognostication and therapeutic decision making. JAMA. 2000; 284(7):835-842. [PubMed: 10938172]

15. Granger CB, Goldberg RJ, Dabbous O, et al. Predictors of hospital mortality in the Global Registry of Acute Coronary Events. Archives of Internal Medicine. 2003; 163(19):2345-2353. [PubMed: 14581255] 
16. Singh SM, FitzGerald G, Yan AT, et al. High-grade atrioventricular block in acute coronary syndromes: insights from the Global Registry of Acute Coronary Events. Eur Heart J. 2014:ehu357.

17. Dauerman HL, Goldberg RJ, White K, et al. Revascularization, stenting, and outcomes of patients with acute myocardial infarction complicated by cardiogenic shock. The American Journal of Cardiology. 2002; 90(8):838-842. [PubMed: 12372570]

18. Piccini JP, White JA, Mehta RH, et al. Sustained Ventricular Tachycardia and Ventricular Fibrillation Complicating Non-ST-Segment Elevation Acute Coronary Syndromes. Circulation. 2012 CIRCULATIONAHA. 111.071860.

19. Halpern NA, Pastores SM. Critical care medicine in the United States 2000-2005: An analysis of bed numbers, occupancy rates, payer mix, costs*. Critical care medicine. 2010; 38(1):65-71. [PubMed: 19730257]

20. Stolker JM, Badawi O, Spertus JA, et al. Intensive care units with low versus high volume of myocardial infarction: clinical outcomes, resource utilization, and quality metrics. Journal of the American Heart Association. 2015; 4(6):e001225. [PubMed: 26066030] 


\section{KEY POINTS}

\section{Question}

What are the patterns of ICU utilization for contemporary U.S. patients with NSTEMI?

\section{Findings}

In this retrospective analysis of the ACTION Registry - Get With The Guidelines linked to Medicare claims data, we found that $43 \%$ of initially stable older patients hospitalized with NSTEMI received care in the ICU, with considerable between hospital variability. Compared with high ICU utilization hospitals, hospitals with low or intermediate ICU utilizations rates were only marginally more selective of higher risk patients for ICU admission, and there was no association between hospital-level ICU utilization and 30day mortality.

\section{Relevance}

ICU utilization for older NSTEMI patients is common and varies substantially among hospitals. 


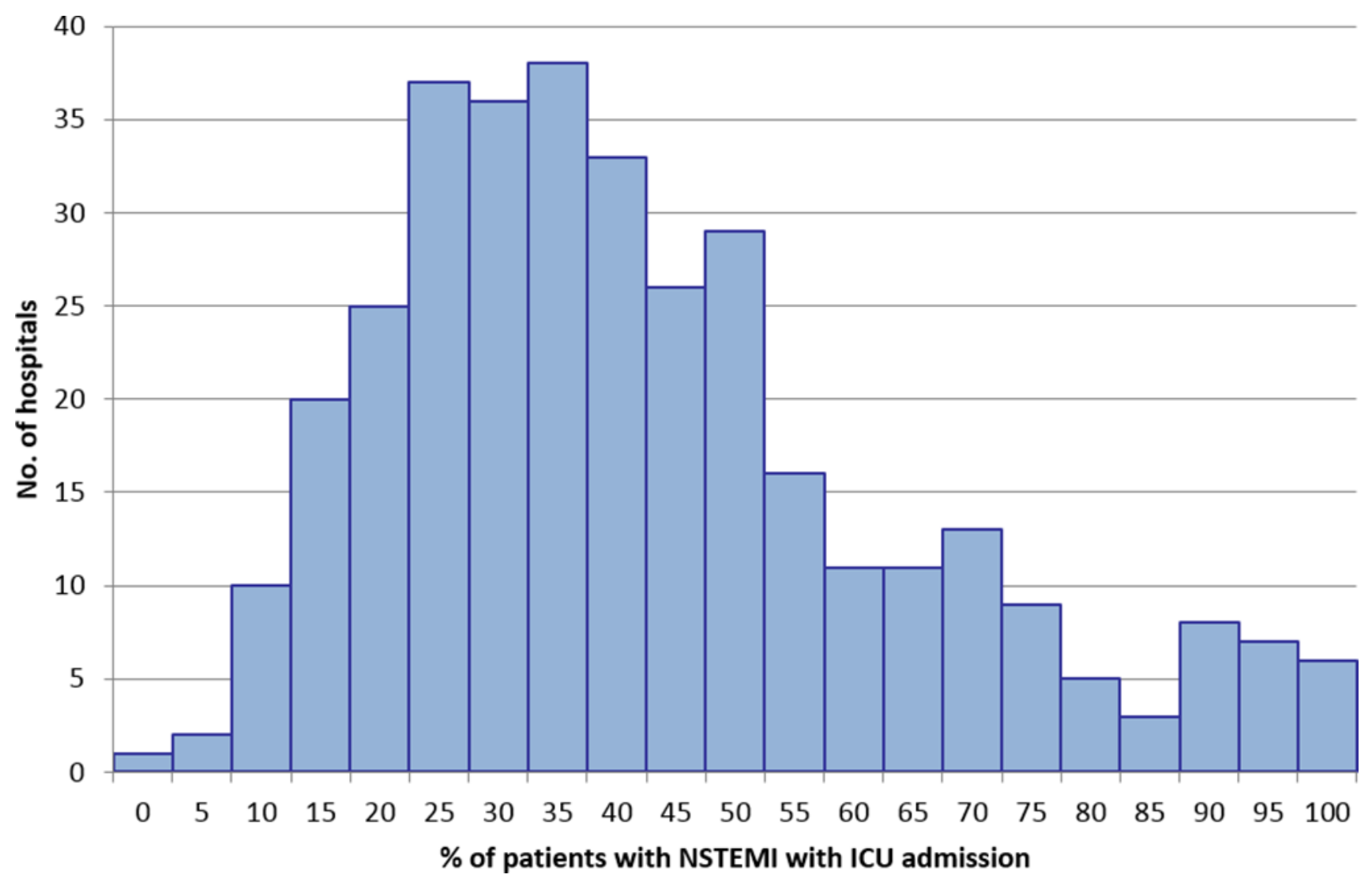

Figure 1.

Inter-hospital variability in ICU utilization for NSTEMI patients

Median $\left(25^{\text {th }}, 75^{\text {th }}\right.$ percentiles $)$ of hospital ICU utilization: $38 \%(26 \%, 54 \%)$. NSTEMI $=$ non-ST segment elevation myocardial infarction; ICU = intensive care unit 


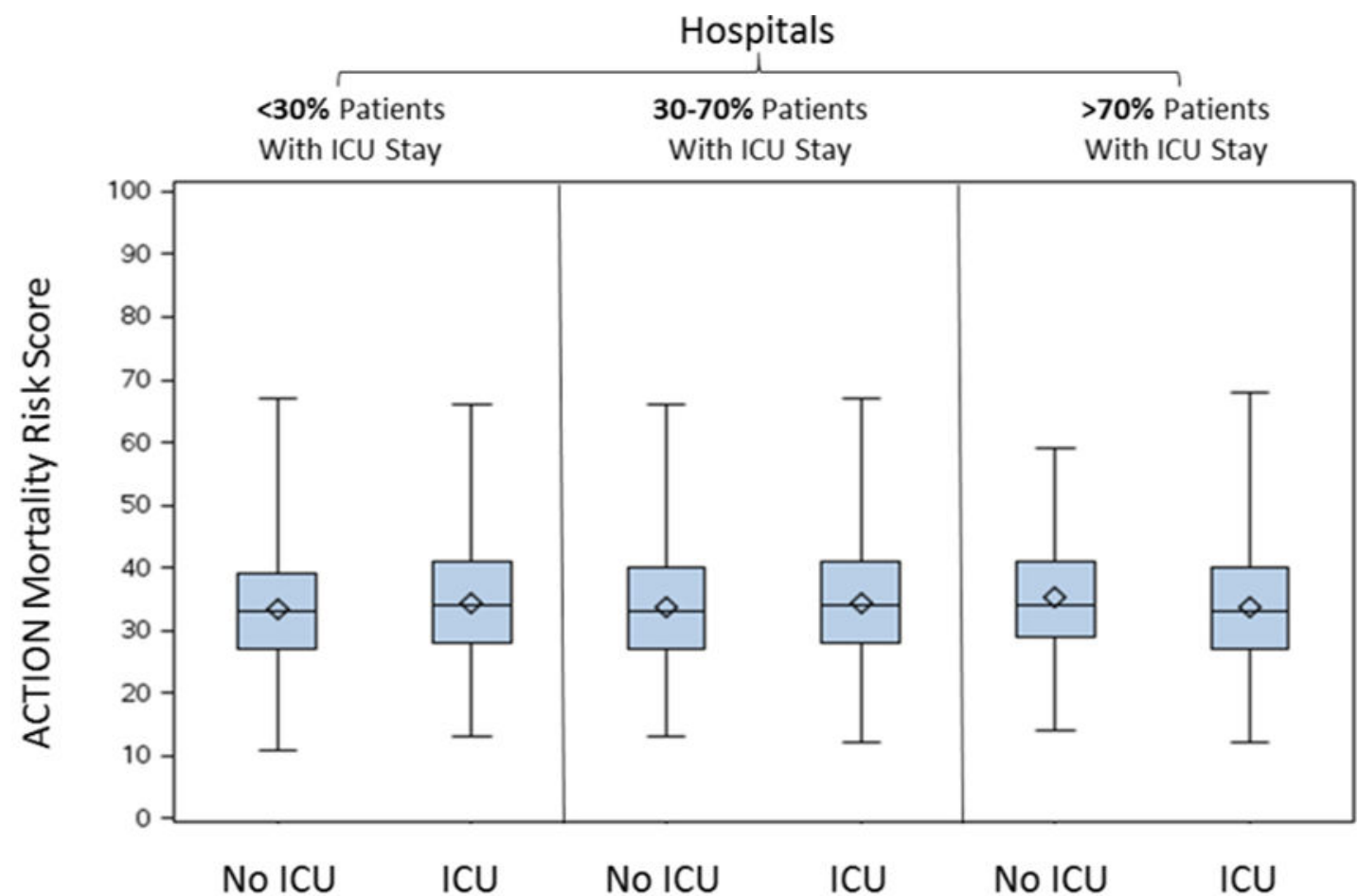

Median ACTION Risk Score
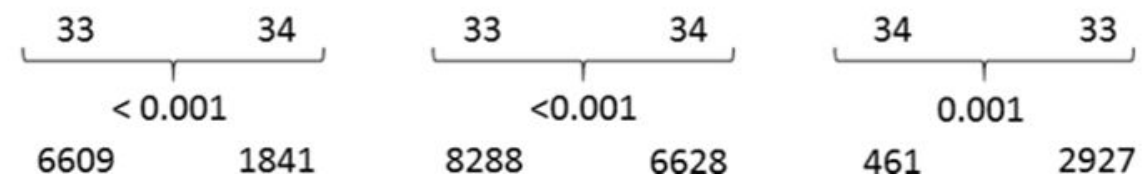

N $\quad 6609 \quad 1841$

8288

6628

461

2927

Figure 2.

Comparison of ACTION risk score between patients treated and not treated in an ICU among hospitals with A) $<30 \%$ of patients with ICU stay, B) 30-70\% of patients with ICU stay, and C) $>70 \%$ of patients with ICU stay 

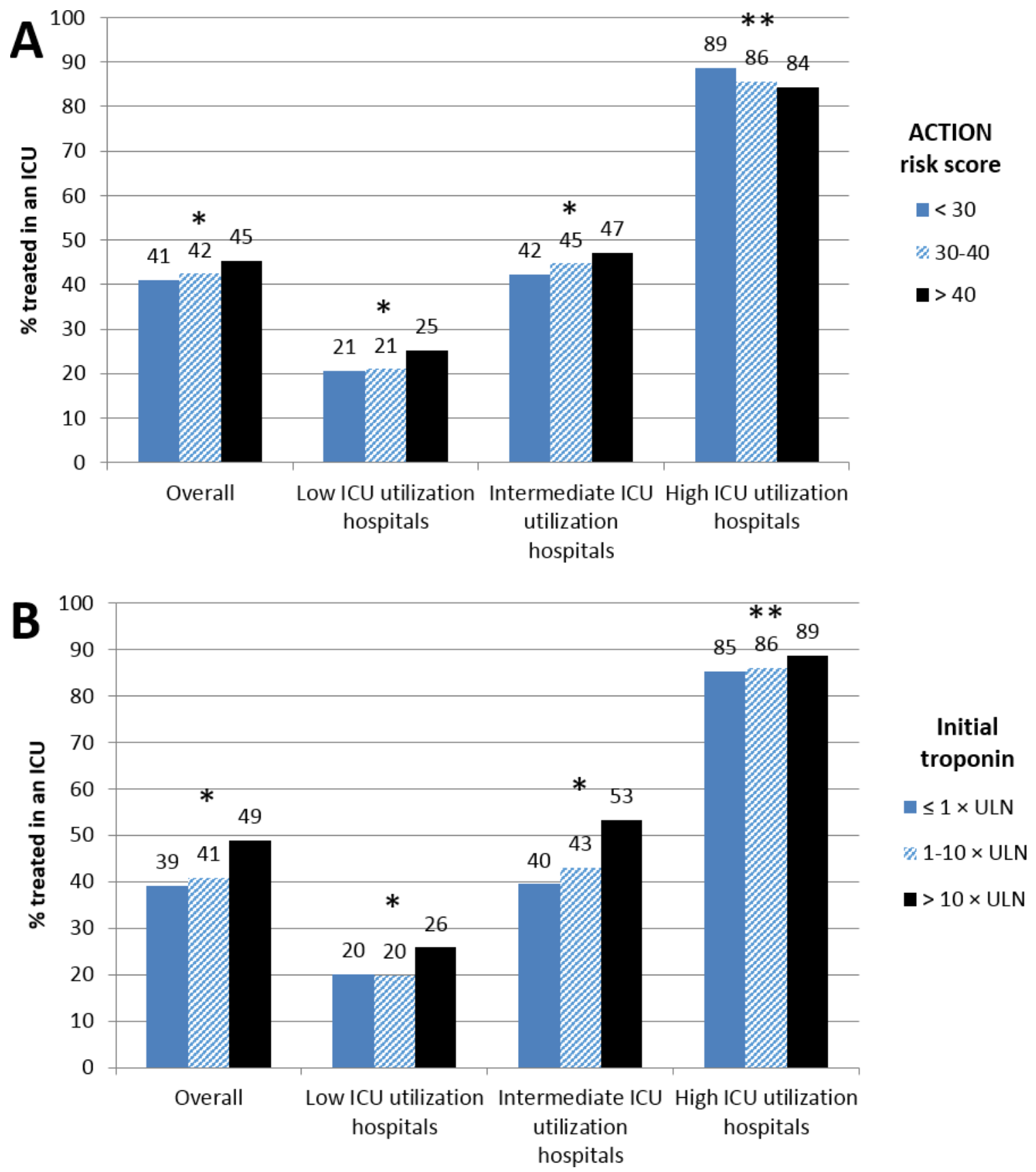

Figure 3.

Proportion of patients treated in an ICU stratified by A) predicted mortality using ACTION risk score, and $\mathrm{B}$ ) initial troponin levels

For panel A, ${ }^{*} p<0.001 ; * * p=0.02$; for panel $\mathrm{B}, * p<0.001 ; * * p=0.05$ 


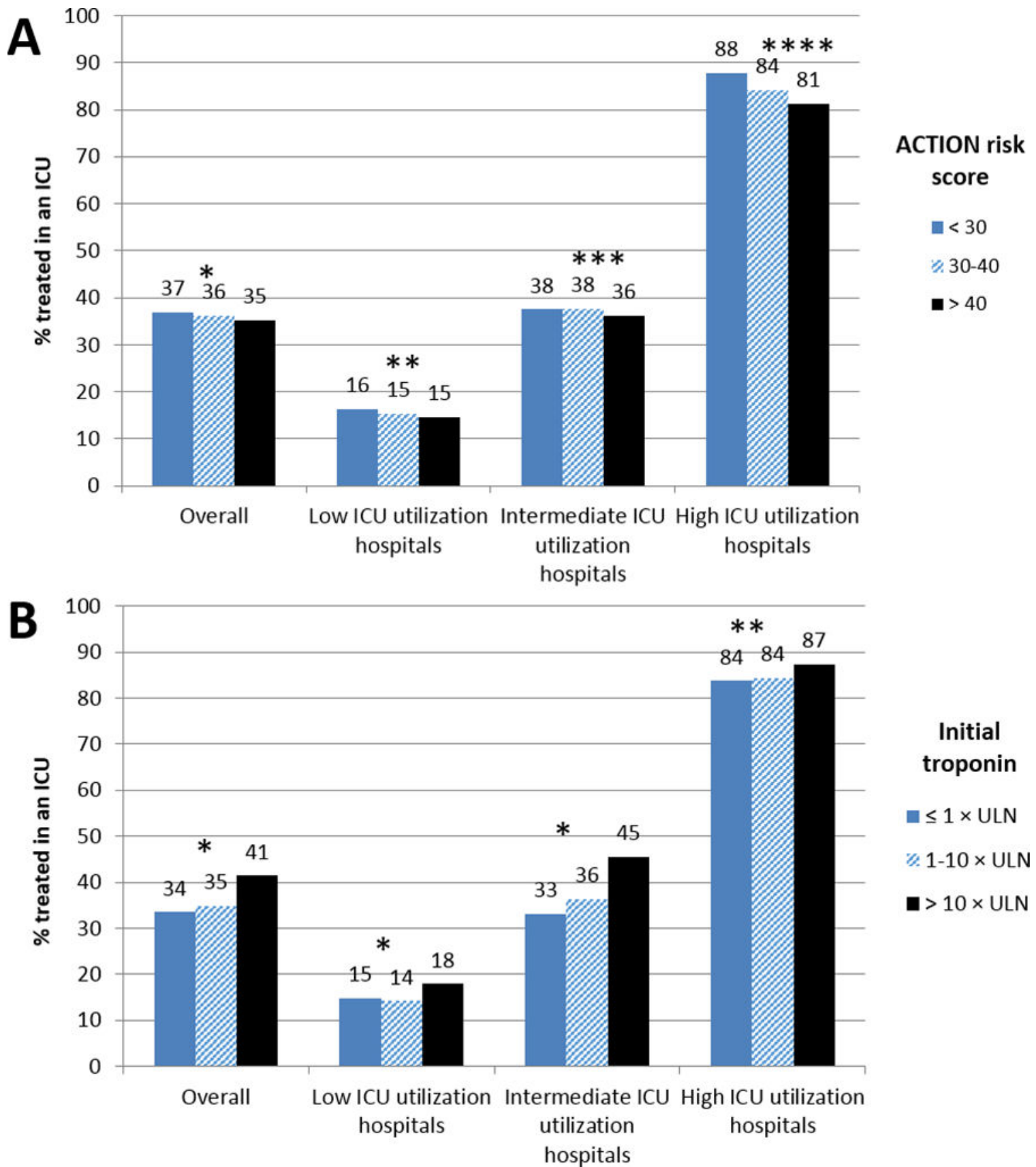

Figure 4.

Proportion of uncomplicated NSTEMI patients treated in an ICU stratified by A) predicted mortality using ACTION risk score, and B) initial troponin values

Uncomplicated patients were defined as those without a complication mandating ICU-level care (in-hospital development of cardiogenic shock, cardiac arrest, or stroke as captured by the ACTION Registry-GWTG data collection form, as well as patients with billed diagnosis or procedure codes indicating hemodynamic compromise requiring medication or mechanical support, arrhythmia requiring defibrillation or pacing, mechanical complication 
post-MI, respiratory failure, stroke, or intracranial hemorrhage). For panel A, * $p=0.19 ; * *$ $p=0.36 ; * * * p=0.45 ; * * * *<<0.001$; for panel $\mathrm{B}, * p<0.001 ; * * p=0.08$ 

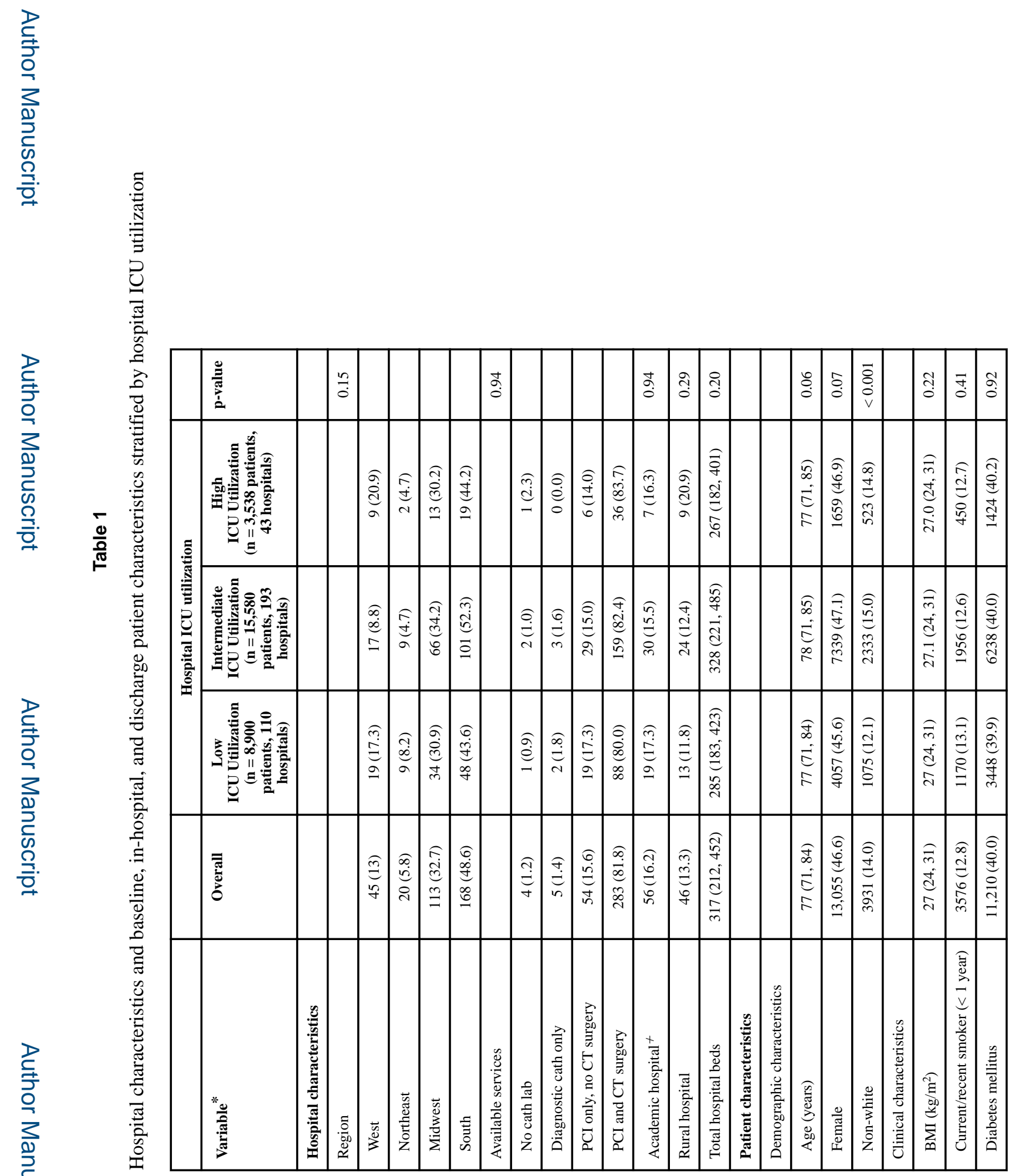

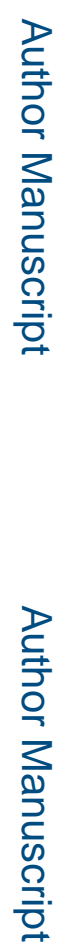

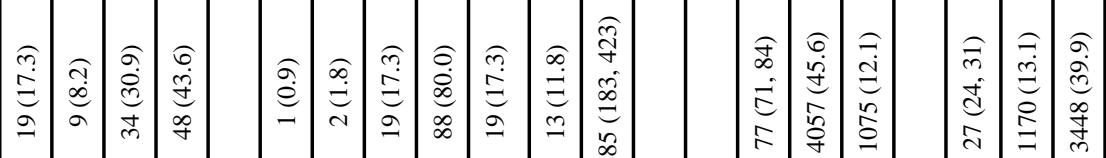




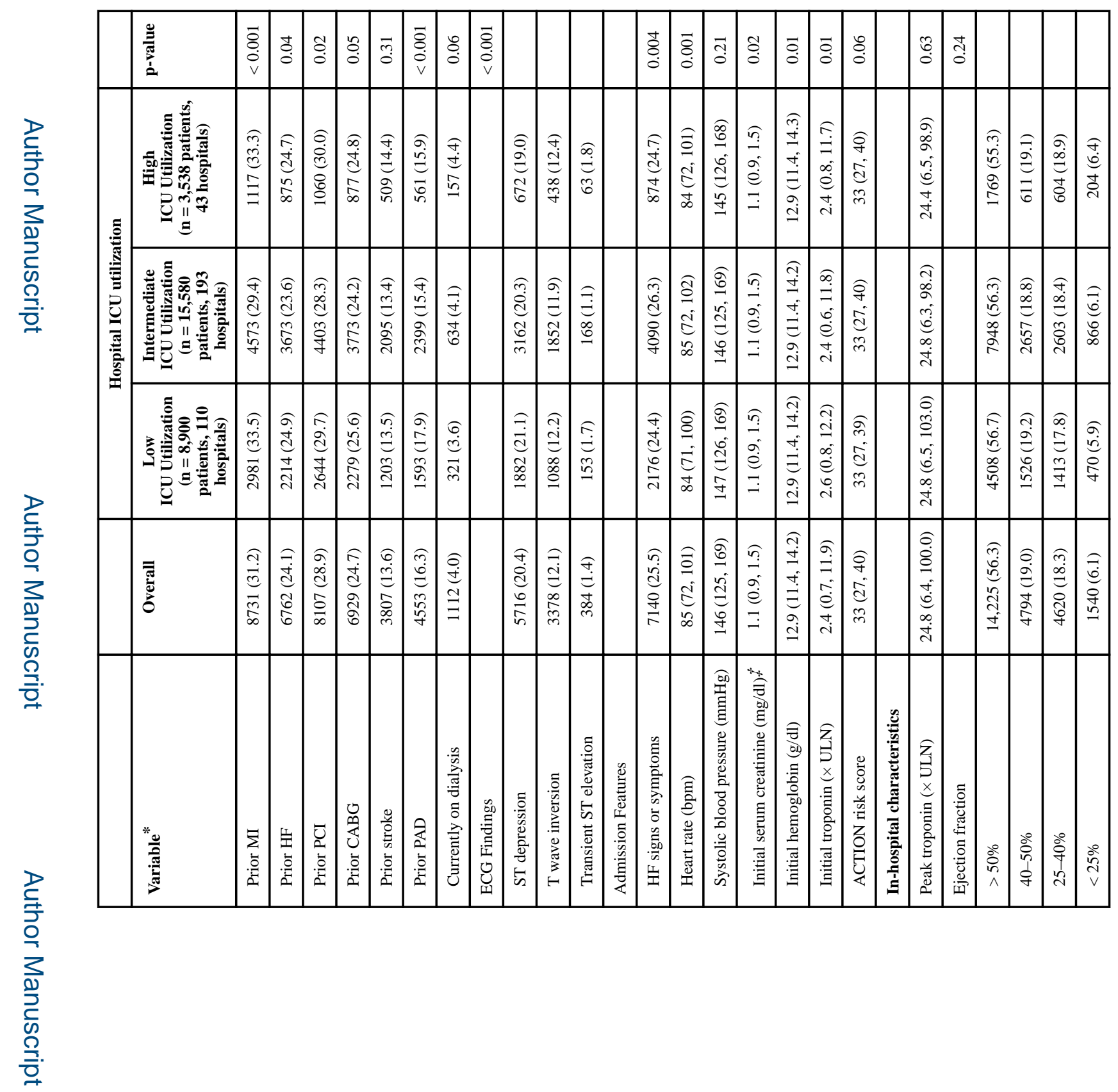

를 


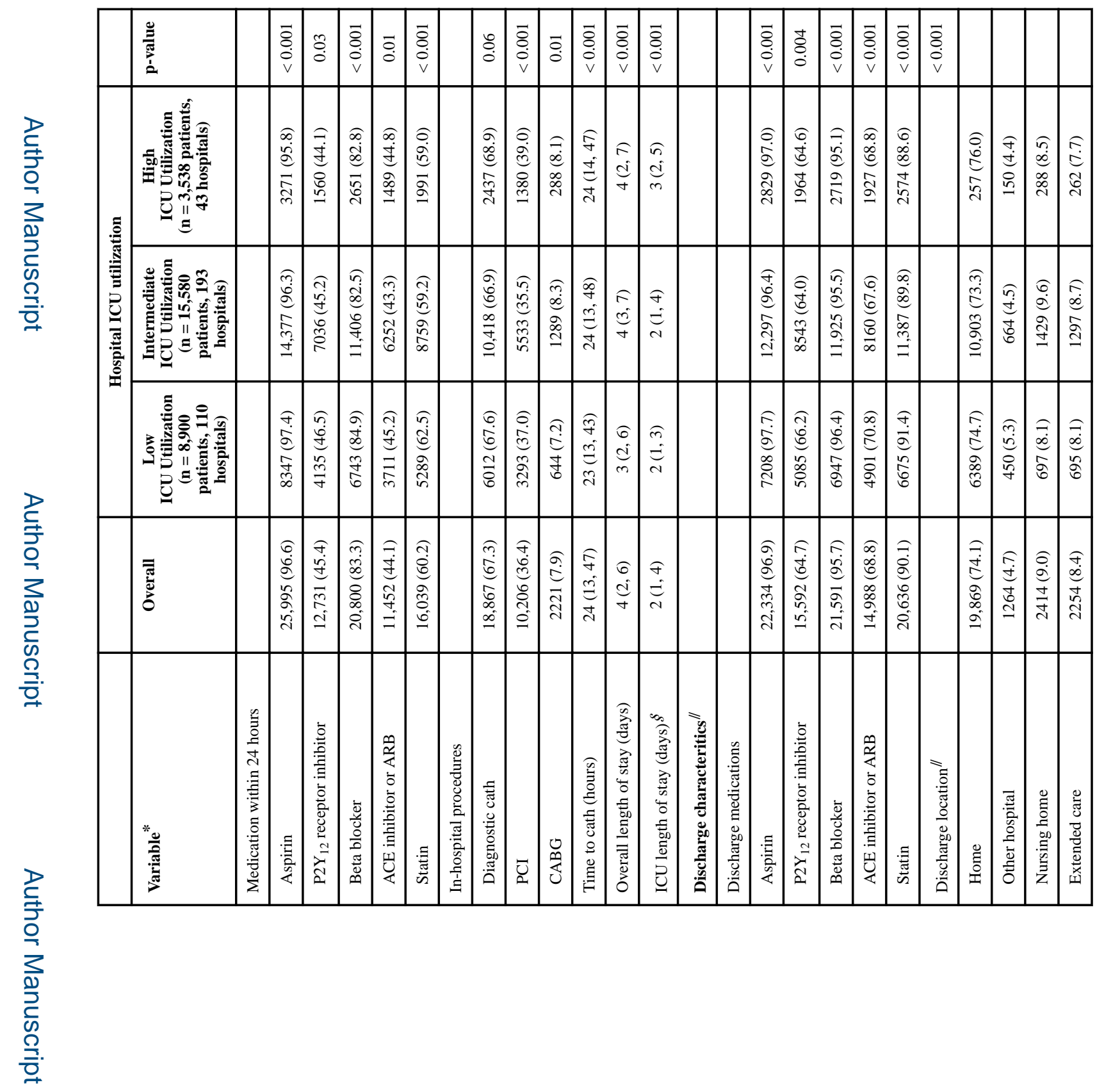

로을 


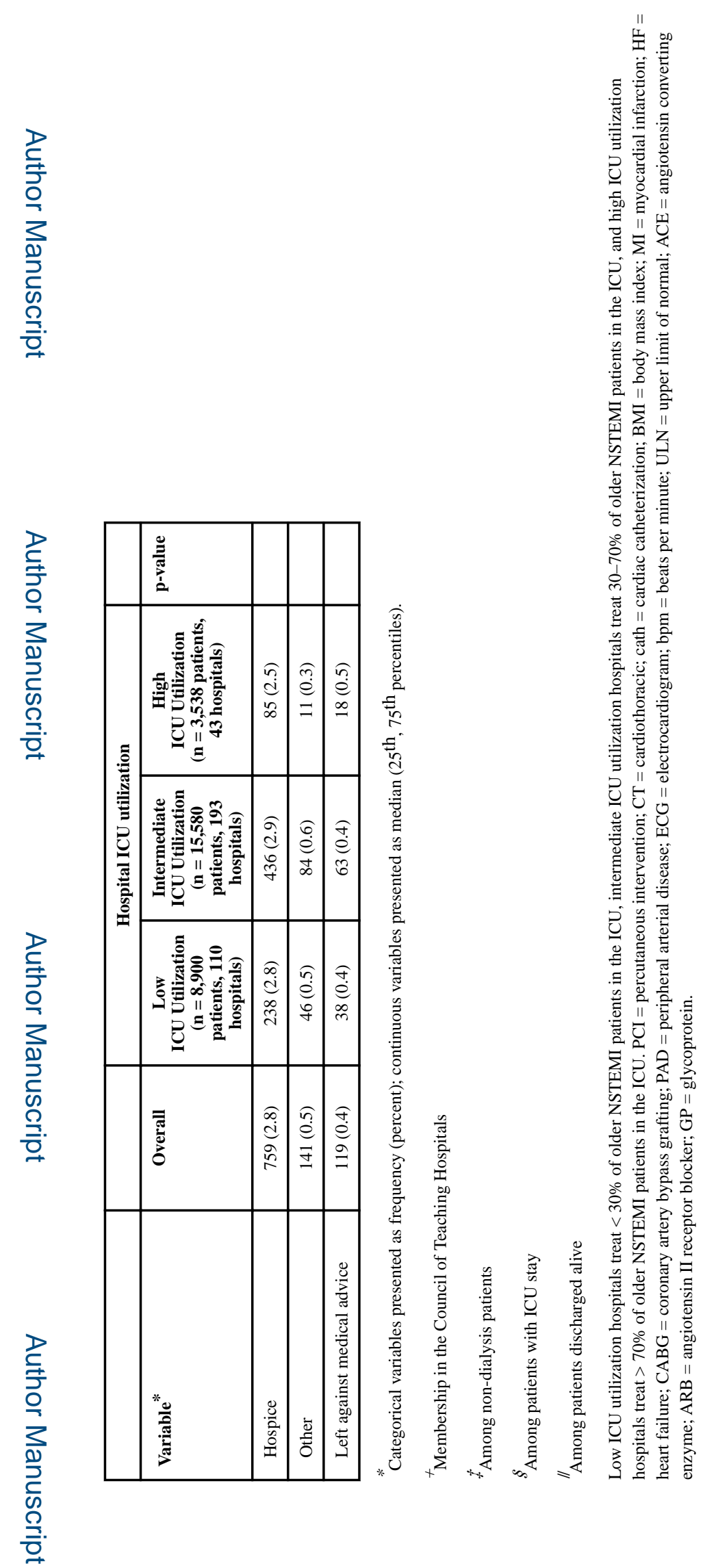

JAMA Cardiol. Author manuscript; available in PMC 2018 January 22. 